JOURNAL OF INTEGRAL EQUATIONS

AND APPLICATIONS

Volume 12, Number 1, Spring 2000

\title{
AN EXPLICIT BOUNDARY INTEGRAL REPRESENTATION OF THE SOLUTION OF THE TWO-DIMENSIONAL HEAT EQUATION AND ITS DISCRETIZATION
}

\author{
I.P. GAVRILYUK AND V.L. MAKAROV
}

\begin{abstract}
An explicit representation of the solution of the two-dimensional heat equation through solutions of boundary integral equations is given. Using this representation we get a semi-discretization, in time, where a sequence of boundary integral equations must be solved. For the last ones the collocation quadrature method can be used.
\end{abstract}

1. Introduction. The horizontal line method, Rothe's method, is often used in order to solve the heat equation numerically. This method consists of a time discretization by finite differences and leads to a sequence of boundary value problems for an inhomogeneous elliptic equation. The last ones can be solved using finite element, finite difference or other methods. In order to overcome difficulties caused by a complicated geometry of the problem the method of integral equations is often used where volume potentials due to the inhomogeneity are incorporated. The advantages of the boundary integral equation method, such as the dimension reduction, the simplification of the geometry of the problem in two-dimensional case, possibility of solving of exterior problems, etc., can be preserved by using approaches involving only boundary integrals, see $[\mathbf{3}, \mathbf{8}]$ and references therein, which leads to very effective discretizations especially in cases of a complicated geometry. The kernels of the boundary integral equations in [8] are evaluated by the Cauchy integral formula and numerical integration in the complex plane. A more explicit procedure for the computation of the kernels involved in the sequence of boundary integral equations and based on some recurrence equations is given in [3]. A drawback of the line method is a fixed convergence order independent of the smoothness of the initial data.

The aims of this paper are to overcome this difficulty and to give an explicit representation of the solution of the two-dimensional heat

\footnotetext{
Received by the editors on October 12, 1998, and in revised form on June 23, 1999.

Copyright (C)2000 Rocky Mountain Mathematics Consortium
} 
equation through solutions of boundary integral equations in the case of stationary boundary conditions. Then using this representation we get a semi-discretization, in time, with accuracy depending on the smoothness of the initial data where a sequence of boundary integral equations must be solved. Our approach is based on the results of $[\mathbf{1}, \mathbf{4}]$ and is different from Rothe's method $[\mathbf{3}]$ and the operational quadrature method [8]. The kernels are explicitly given by wellstudied classical special functions. A fully discrete method can then be constructed analogously to $[\boldsymbol{3}]$. We also give an approximation with spectral accuracy for a time-dependent boundary condition using Duhamel's integrals. It allows us to use the previous results concerning the stationary boundary conditions.

\section{The heat equation with time-independent boundary} condition. Let us consider the following problem

$$
\begin{aligned}
\frac{\partial u}{\partial t} & =\Delta u(x, t), \quad(x, t) \in D \times(0, T], \\
u(x, t) & =\phi(x), \quad x \in \Gamma, \\
u(x, 0) & =0, \quad x \in D .
\end{aligned}
$$

Homogeneous boundary conditions are often more convenient from various points of view. In order to deal with such boundary conditions, we represent the solution of (2.1) as

$$
u(x, t)=v(x, t)+V(x)
$$

where the functions $v$ and $V$ satisfy the following equations

$$
\begin{aligned}
\Delta V(x) & =0, \quad x \in D, \\
V(x) & =\phi(x), \quad x \in \Gamma,
\end{aligned}
$$

and

$$
\begin{aligned}
\frac{\partial v}{\partial t} & =\Delta v(x, t), \quad(x, t) \in D \times(0, T], \\
v(x, t) & =0, \quad x \in \Gamma, \\
v(x, 0) & =-V(x), \quad x \in D .
\end{aligned}
$$


We define the self-adjoint operator $A$ by

$$
D(A)=H^{2}(D) \cap \stackrel{\circ}{H}^{1}(D), \quad A u=-\Delta u \quad \forall u \in D(A)
$$

and denote its range by $R(A)$, where $H^{l}(D), \stackrel{\circ}{H}^{l}(D)$ state for the wellknown Sobolev spaces. Then the problem (2.4) can be written in the operator form

$$
\frac{d v(t)}{d t}+A v=0, \quad v(0)=-V,
$$

where $v(t)$ is a vector-valued function.

Using the results of $[\mathbf{1}, \mathbf{4}]$, we get

$$
v(x, t)=e^{-\gamma t} \sum_{n=0}^{\infty}(-1)^{n} L_{n}^{(0)}(2 \gamma t) w_{n}(x),
$$

where $\gamma$ is an arbitrary positive number, $L_{n}^{(0)}(\xi)$ is the Laguerre polynomial of degree $n$ and $w_{n}$ satisfies the equations

$$
(\gamma I+A) w_{n+1}=(\gamma I-A) w_{n}, \quad n=0,1, \ldots
$$

or

$$
\begin{aligned}
(\gamma-\Delta) w_{n+1}(x) & =(\gamma+\Delta) w_{n}(x), \quad x \in D, n=0,1, \ldots, \\
w_{n+1}(x) & =0, \quad x \in \Gamma .
\end{aligned}
$$

For $w_{0}$ we have

$$
w_{0}=-\left[I+T_{\gamma}(A)\right] V=-2 \gamma(\gamma+A)^{-1} V,
$$

where $T_{\gamma}(A)=(\gamma I-A)(\gamma I+A)^{-1}$ is the Cayley transform of the operator $A$. We denote by $S_{\gamma}: V \rightarrow u$ the (boundary) operator defined by

$$
\begin{aligned}
(\gamma-\Delta) u(x) & =0, \quad x \in D, \\
u(x) & =-V(x), \quad x \in \Gamma,
\end{aligned}
$$

and set $P_{\gamma}=I-S_{\gamma}$. Then we can write

$$
\begin{aligned}
(\gamma-\Delta) w_{0}(x) & =-2 \gamma V(x), \quad x \in D, \\
w_{0}(x) & =0, \quad x \in \Gamma,
\end{aligned}
$$


or, similarly,

$$
w_{0}=-2 P_{\gamma}(V)
$$

As a natural approximation for the exact solution (2.5) one can use the truncated sum

$$
v^{N}(x, t)=e^{-\gamma t} \sum_{n=0}^{N}(-1)^{n} L_{n}^{(0)}(2 \gamma t) w_{n}(x) .
$$

It was shown in $[\mathbf{1}, \mathbf{4}]$ that

$$
\left\|v(\cdot, t)-v^{N}(\cdot, t)\right\| \leq c N^{-\sigma}\left\|A^{\sigma} w_{0}\right\|
$$

provided that $w_{0} \in D\left(A^{\sigma}\right)$ for some $\sigma>0$. The truncated sum (2.10) together with (2.6) can be considered as a semi-discrete approximation for the problem (2.4). The estimate (2.11) shows that this approximation possesses at least a convergence order $O\left(N^{-1}\right)$ provided that $V \in R(A)$.

The next lemma shows that all $w_{n}, n=1,2, \ldots$, can be represented using the operator $S_{\gamma}$.

Lemma 2.1. The functions $w_{n}(x), x \in D$, can be represented through the boundary operator $S_{\gamma}$ by the formulas

$$
w_{0}=-2 P_{\gamma}(V), w_{n}=-2 P_{\gamma}\left[V-\frac{(-2 \gamma)^{n}}{n !} \frac{\partial^{n}}{\partial \gamma^{n}} S_{\gamma}(V)\right], \quad n \geq 1
$$

Proof. Using the fact that $\Delta^{k} V=0, k=1,2, \ldots$, we get

$$
\begin{aligned}
(\gamma-\Delta)^{n} w_{n}(x) & =(\gamma+\Delta)^{n} w_{0} \\
& =(2 \gamma-\gamma+\Delta)^{n} w_{0} \\
& =\sum_{p=0}^{n}\left(\begin{array}{l}
n \\
p
\end{array}\right)(2 \gamma)^{n-p}(-\gamma+\Delta)^{p} w_{0} \\
& =-2 \sum_{p=1}^{n}\left(\begin{array}{l}
n \\
p
\end{array}\right)(2 \gamma)^{n-p}(-\gamma)^{p} V-(2 \gamma)^{n} 2\left[V-S_{\gamma}(V)\right] \\
& =-2 V\left[(2 \gamma-\gamma)^{n}-(2 \gamma)^{n}\right]-(2 \gamma)^{n} 2\left[V-S_{\gamma}(V)\right] \\
& =-2\left[\gamma^{n} V-(2 \gamma)^{n} S_{\gamma}(V)\right], \quad x \in D, \\
w_{n}(x) & =0, \quad x \in \Gamma .
\end{aligned}
$$


In order to solve (2.13) we use the following result which one can get analogously to $[\mathbf{1 0}]$ : if

$$
\begin{aligned}
(\gamma-\Delta)^{n} u & =v(x), \quad x \in D, \\
u(x) & =0, \quad x \in \Gamma
\end{aligned}
$$

and $(\gamma-\Delta) v=0$, then

$$
u=\frac{(-1)^{n}}{n !} P_{\gamma} \frac{\partial^{n} v}{\partial \gamma^{n}}
$$

Representing $w_{n}=w_{n_{1}}+w_{n_{2}}$, where $w_{n_{1}}, w_{n_{2}}$ are the solutions of the problems

$$
\begin{aligned}
& (\gamma-\Delta)^{n} w_{n_{1}}(x)=-2 \gamma^{n} V(x), \quad x \in D, \quad w_{n_{1}}(x)=0, \quad x \in \Gamma, \\
& (\gamma-\Delta)^{n} w_{n_{2}}(x)=2(2 \gamma)^{n} S_{\gamma}(V), \quad x \in D, \quad w_{n_{2}}(x)=0, \quad x \in \Gamma
\end{aligned}
$$

we get

$$
w_{n_{1}}=-2 P_{\gamma}(V), \quad w_{n_{2}}=2(2 \gamma)^{n} \frac{(-1)^{n}}{n !} P_{\gamma} \frac{\partial^{n}}{\partial \gamma^{n}} S_{\gamma}(V)
$$

i.e.,

$$
w_{n}=-2 P_{\gamma}\left[V-\frac{(-2 \gamma)^{n}}{n !} \frac{\partial^{n}}{\partial \gamma^{n}} S_{\gamma}(V)\right],
$$

which proves the lemma.

Note that the formula (2.12) allows us to avoid volume potentials when solving the inhomogeneous equations (2.6). Using the volume potentials has the drawback of destroying the two main advantages of the boundary integral equation method: this method reduces the dimensionality of the problem and is especially useful for unbounded domains since a problem over an unbounded domain is replaced by one over a boundary. The solution of (2.8) can be represented as the following double layer potential

$$
S_{\gamma}(V)(x)=\frac{1}{\pi} \int_{\Gamma} \frac{\partial K_{0}(\sqrt{\gamma}|x-y|)}{\partial \nu(y)} g(y) d s(y),
$$


where $g(y)$ is an unknown density, $\nu(y)$ is the outer normal to $\Gamma$, $|x-y|$ is the distance between the points $x$ and $y, K_{n}(z), n=0,1, \ldots$, denotes the modified Bessel function of order $n$, see, e.g., $[\mathbf{2}, \mathbf{7}, \mathbf{1 1}$, 12], computer algebra tools MATHEMATICA, MAPLE, REDUCE or World Wide Web: [5],

$$
\begin{aligned}
& K_{0}(z)=-I_{0}(z) \ln \frac{z}{2}+\sum_{m=0}^{\infty}\left(\frac{z}{2}\right)^{2 m} \frac{\psi(m+1)}{(m !)^{2}} \\
& K_{n}(z)=(-1)^{n+1} I_{n}(z) \ln \left(\frac{z}{2}\right)+\frac{1}{2} \sum_{m=0}^{n-1}(-1)^{m}\left(\frac{z}{2}\right)^{2 m-n} \frac{(n-m-1) !}{m !} \\
&+\frac{(-1)^{n}}{2} \sum_{m=0}^{\infty}\left(\frac{z}{2}\right)^{n+2 m} \frac{\psi(n+m+1)+\psi(m+1)}{m !(n+m) !} \\
& n=1,2, \ldots,
\end{aligned}
$$

$\psi(z)$ is the logarithmic derivative of the gamma function, $\psi(m+1)=1+$ $(1 / 2)+\cdots+(1 / m)-\gamma, \gamma=\lim _{m \rightarrow \infty}\left(\sum_{n=1}^{m}(1 / n)-\ln m\right)=0.57721 \ldots$ is the Euler's constant, and

$$
I_{n}(z)=\sum_{m=0}^{\infty} \frac{(z / 2)^{2 m+n}}{m ! \Gamma(m+n+1)}
$$

is the Bessel function of the complex argument of order $n$.

The density $g(y)$ is the solution of the boundary integral equation of the second kind

$$
-g(x)+\frac{1}{\pi} \int_{\Gamma} \frac{\partial K_{0}(\sqrt{\gamma}|x-y|)}{\partial \nu(y)} g(y) d s(y)=-V(x), \quad x \in \Gamma .
$$

The operator $S_{\gamma}$ can also be represented through the single layer potential

$$
S_{\gamma}(\varphi)(x)=-\frac{1}{\pi} \int_{\Gamma} K_{0}(\sqrt{\gamma}|x-y|) \tilde{g}(y) d s(y), \quad x \in D,
$$

where the density $\tilde{g}(y)$ satisfies the boundary integral equation of the first kind

$$
\frac{1}{\pi} \int_{\Gamma} \tilde{g}(y) K_{0}(\sqrt{\gamma}|x-y|) d s(y)=-V(x), \quad x \in \Gamma .
$$


Let us denote

$$
\begin{aligned}
\Phi_{n}(x, y) & \equiv \Phi_{n}(\sqrt{\gamma}|x-y|)=\frac{(-2 \gamma)^{n}}{n !} \frac{\partial^{n}}{\partial \gamma^{n}} K_{0}(\sqrt{\gamma}|x-y|), \\
g^{(j)}(y) & \equiv g^{(j)}(y, \gamma)=\frac{(-2 \gamma)^{j}}{j !} \frac{\partial^{j}}{\partial \gamma^{j}} g(y, \gamma) .
\end{aligned}
$$

By the differentiation of (2.14) and (2.17), we get

$$
\begin{gathered}
\frac{(-2 \gamma)^{n}}{n !} \frac{\partial^{n}}{\partial \gamma^{n}} S_{\gamma}(V)=\frac{1}{\pi} \int_{\Gamma} \sum_{\substack{j=0 \\
x}}^{n} \frac{\partial}{\partial \nu(y)} \Phi_{n-j}(x, y) g^{(j)}(y) d s(y), \\
\end{gathered}
$$

$$
\begin{gathered}
-g^{(j)}(x, \gamma)+\frac{1}{\pi} \int_{\Gamma} \frac{\partial}{\partial \nu(y)} K_{0}(\sqrt{\gamma}|x-y|) g^{(j)}(y, \gamma) d s(y) \\
=-\frac{1}{\pi} \int_{\Gamma} \sum_{l=0}^{j-1} \frac{\partial}{\partial \nu(y)} \Phi_{j-l}(x, y) g^{(l)}(y, \gamma) d s(y), \\
x \in \Gamma, j=0,1,2, \ldots,
\end{gathered}
$$

where the righthand side in (2.21) is equal to $-V(x)$ for $j=0$.

Analogously we get by the differentiation of (2.18) and (2.19),

$$
\begin{aligned}
\frac{(-2 \gamma)^{n}}{n !} \frac{\partial^{n}}{\partial \gamma^{n}} S_{\gamma}(V) & =\frac{1}{\pi} \int_{\Gamma} \sum_{j=0}^{n} \Phi_{n-j}(x, y) \tilde{g}^{(j)}(y) d s(y), \quad x \in D, \\
(2.23) \frac{1}{\pi} \int_{\Gamma} K_{0}(\sqrt{\gamma}|x-y|) \tilde{g}^{(j)}(y, \gamma) d s(y) & \\
& =-\frac{1}{\pi} \int_{\Gamma} \sum_{l=0}^{j-1} \Phi_{j-l}(x, y) \tilde{g}^{(l)}(y, \gamma) d s(y), \quad x \in \Gamma,
\end{aligned}
$$

$j=0,1,2, \ldots$, where the righthand side is equal to $-V(x)$ for $j=0$. 
The equation (2.21) takes the form

$$
\begin{aligned}
-g^{(j)}(x, \gamma)+\frac{1}{\pi} \int_{\Gamma} \frac{\partial}{\partial \nu(y)} K_{0}(\sqrt{\gamma}|x-y|) g^{(j)}(y, \gamma) d s(y) \\
=-g^{(j-1)}(x, \gamma) \\
\quad+\frac{1}{\pi} \int_{\Gamma} \sum_{l=0}^{j-1} \frac{\partial}{\partial \nu(y)}\left[\Phi_{j-1-l}(x, y)-\Phi_{j-l}(x, y)\right] g^{(l)}(y, \gamma) d s(y) .
\end{aligned}
$$

Using the known result $[2]$

$$
\left(\frac{d}{z d z}\right)^{m} K_{0}(z)=(-1)^{m} z^{-m} K_{m}(z)
$$

and setting $z^{2}=2 \xi, z=\sqrt{2 \xi}$, we get

$$
\left(\frac{d}{d \xi}\right)^{m} K_{0}(\sqrt{2 \xi})=(-1)^{m}(2 \xi)^{-m / 2} K_{m}(\sqrt{2 \xi})
$$

which further yields for $\xi=\gamma|x-y|^{2} / 2, \gamma=2 \xi|x-y|^{-2}$,

$$
\Phi_{n}(x, y)=\frac{(-2 \gamma)^{n}}{n !} \frac{\partial^{n}}{\partial \xi^{n}} K_{0}(\sqrt{2 \xi})=\frac{1}{n !}(\sqrt{\gamma}|x-y|)^{n} K_{n}(\sqrt{\gamma}|x-y|) .
$$

The function $K_{n}(z)$ can be expressed through only two functions $K_{0}(z)$ and $K_{0}^{\prime}(z)$. Actually, using the formulas [2]

$$
\begin{aligned}
& K_{\nu-1}(z)-K_{\nu+1}(z)=-2 \nu z^{-1} K_{\nu}(z), \\
& K_{\nu-1}(z)+K_{\nu+1}(z)=-2 K_{\nu}^{\prime}(z),
\end{aligned}
$$

setting $i z$ for $z$ in $(2.26)$ and denoting $i^{\nu} K_{\nu}(i z)=\tilde{K}_{\nu}(z)$ we get

$$
\tilde{K}_{\nu-1}(z)+\tilde{K}_{\nu+1}(z)=\frac{2 \nu}{z} \tilde{K}_{\nu}(z),
$$

which yields

$$
\tilde{K}_{m+1}(z)=\tilde{K}_{1}(z) R_{m, 1}(z)-\tilde{K}_{0}(z) R_{m-1,2}(z),
$$


where $R_{m, \nu}(z)$ denotes the Lommel's polynomial in $1 / z$

$$
\begin{aligned}
R_{m, \nu}(z) & =\sum_{n=0}^{\lfloor m / 2\rfloor} \frac{(-1)^{m}(m-n) !(\nu+m-n-1) !}{n !(m-2 n) !(\nu+n-1) !}\left(\frac{z}{2}\right)^{-m+2 n} \\
& =\frac{\Gamma(\nu+m)}{\Gamma(\nu)}\left(\frac{z}{2}\right)^{-m}{ }_{2} F_{3}\left(\frac{1-m}{2},-\frac{m}{2} ; \nu,-m, 1-\nu-m ;-z^{2}\right)
\end{aligned}
$$

and ${ }_{p} F_{q}$ is the generalized hypergeometric function. One can find various formulas, properties for Bessel's functions and Lommel's polynomials in $[2,5,7, \mathbf{1 1}, \mathbf{1 2}]$.

It follows from (2.28) that

$$
K_{n}(z)=(-i)^{n-1}\left[-K_{0}^{\prime}(z) R_{n-1,1}(-i z)+i K_{0}(z) R_{n-2,2}(-i z)\right]
$$

and we get from $(2.25)$

$$
\begin{aligned}
& \Phi_{n}(x, y) \\
& \quad=\frac{(-i)^{n-1}}{n !}\left[K_{0}(\sqrt{\gamma}|x-y|) v_{n}(\sqrt{\gamma}|x-y|)-K_{0}^{\prime}(\sqrt{\gamma}|x-y|) \tilde{v}_{n}(\sqrt{\gamma}|x-y|)\right],
\end{aligned}
$$

where

$$
\begin{gathered}
v_{n}(z)=i z^{n} R_{n-2,2}(-i z), \quad \tilde{v}_{n}(z)=z^{n} R_{n-1,1}(-i z), \\
R_{-1,1}=0, \quad R_{0,1}=1, \quad R_{-2,2}=-1, \quad R_{-1,2}=0 \\
n=0,1,2, \ldots
\end{gathered}
$$

One can see from (2.29) and (2.32) that $v_{n}(z)$ is an even and $\tilde{v}_{n}(z)$ is an odd polynomial in $z=\sqrt{\gamma}|x-y|$ of degree $\leq n$. Analogously as above one can also find

$$
\frac{\partial \Phi_{n}(x, y)}{\partial y_{i}}=\frac{\gamma}{n !}\left(x_{i}-y_{i}\right)(\sqrt{\gamma}|x-y|)^{n-1} K_{n-1}(\sqrt{\gamma}|x-y|)
$$

and following one can find $\left(\partial \Phi_{n}(x, y) / \partial \nu(y)\right)$.

Summarizing these results together with Lemma 2.1, we arrive at the following statement. 
Theorem 2.1. The functions $w_{n}(x), x \in D$ from (2.5) and (2.10) can be represented by the following formulas

$$
w_{n}(x)=-2\left[\tilde{V}^{(1)}(x)-\frac{1}{\pi} \int_{\Gamma} \frac{\partial K_{0}(\sqrt{\gamma}|x-y|)}{\partial \nu(y)} \hat{g}^{(0)}(y) d s(y)\right]
$$

or

$$
w_{n}(x)=-2\left[\tilde{V}^{(2)}(x)+\frac{1}{\pi} \int_{\Gamma} K_{0}(\sqrt{\gamma}|x-y|) \hat{\tilde{g}}^{(0)}(y) d s(y)\right], \quad x \in D,
$$

where

$$
\begin{aligned}
& \tilde{V}^{(1)}(x)=V(x)-\frac{1}{\pi} \int_{\Gamma} \sum_{j=0}^{n} \frac{\partial}{\partial \nu(y)} \Phi_{n-j}(x, y) g^{(j)}(y) d s(y), \quad x \in D, \\
& \tilde{V}^{(2)}(x)=V(x)-\frac{1}{\pi} \int_{\Gamma} \sum_{j=0}^{n} \Phi_{n-j}(x, y) \tilde{g}^{(j)}(y) d s(y), \quad x \in D,
\end{aligned}
$$

$K_{0}(\sqrt{\gamma}|x-y|), \Phi_{n}(x, y),\left(\partial \Phi_{n}(x, y) / \partial \nu(y)\right)$ are given explicitly by (2.15), (2.31), (2.33), $g^{(j)}(y), \tilde{g}^{(j)}(y), j \geq 0$, are the solutions of the boundary integral equations (2.21), (2.23), $\hat{g}^{(0)}(y)$ and $\hat{\tilde{g}}^{(0)}(y)$ are the solutions of the boundary integral equations (2.17), (2.19) with the functions $\tilde{V}^{(1)}$ and $\tilde{V}^{(2)}$ instead of $V$.

Now we are in the position to formulate the following procedure and algorithm for solving the problem (2.1) through boundary integral equations only.

\section{procedure $\operatorname{HEBBIE}\left(V, w_{n}\right)$}

(computes $w_{n}(x)$ by boundary integral equations based on the single layer potential; Input: $V$; Output: $w_{n}$ )

\section{begin}

1. Find $\tilde{g}^{(0)}(y), y \in \Gamma$ as the solution of the boundary integral equation (2.19) with the input-function $V$;

2. For $j=1, \ldots, n$, find $\tilde{g}^{(j)}(y), y \in \Gamma$ as the solution of boundary integral equations (2.23); 
3. Find

$$
\tilde{V}^{(2)}(x)=V(x)-\frac{1}{\pi} \int_{\Gamma} \sum_{j=0}^{n} \Phi_{n-j}(x, y) \tilde{g}^{(j)}(y) d s(y), \quad x \in D
$$

and $\hat{\tilde{g}}^{(0)}(y), y \in \Gamma$, as the solution of the boundary integral equation

$$
\frac{1}{\pi} \int_{\Gamma} \hat{\tilde{g}}(y) K_{0}(\sqrt{\gamma}|x-y|) d s(y)=-\tilde{V}^{(2)}(x), \quad x \in \Gamma ;
$$

4. Find

$$
w_{n}(x)=-2 \tilde{V}^{(2)}(x)-\frac{2}{\pi} \int_{\Gamma} K_{0}(\sqrt{\gamma}|x-y|) \hat{\tilde{g}}^{(0)}(y) d s(y), \quad x \in D
$$

end

The next algorithm computes the approximate solution of the problem (2.1) by (2.10).

\section{Algorithm 2.1.}

(computes the approximate solution of the heat equation with a time independent boundary condition through boundary integral equations using the procedure HEBBIE)

begin

1. For $n=0,1, \ldots, N$

$\operatorname{HEBBIE}\left(V, w_{n}\right)$;

2. Find $v^{N}(x, t)$ by $(2.10)$

end

An algorithm based on the double layer potential can be formulated analogously. 
It is easy to see that

$$
\begin{aligned}
(\gamma-\Delta) \Phi_{n}= & \frac{(-2 \gamma)^{n}}{n !} \frac{\partial^{n}}{\partial \gamma^{n}}(\gamma-\Delta) K_{0}(\sqrt{\gamma}|x-y|) \\
& -\frac{(-2 \gamma)^{n}}{(n-1) !} \frac{\partial^{n-1}}{\partial \gamma^{n-1}} K_{0}(\sqrt{\gamma}|x-y|) \\
= & 2 \gamma \Phi_{n-1} .
\end{aligned}
$$

Using this relation one can derive recurrence equations for the polynomials $v_{n}, \tilde{v}_{n}$ and their coefficients. Comparing (2.34) with (2.5) in [3] we conclude that our method is closely related to the methods from $[\mathbf{3}$, 8] (for the relation between the last two methods, see [3]). However, we wish to point out that we are working with kernels explicitly given through well-studied classical functions whereas the kernels in [3] are evaluated using explicit recurrence formulas analogously to (2.34) and the kernels from [8] are obtained using Cauchy integral formula and numerical integration in the complex plane. Another distinctive feature of our approach is the original time discretization (2.6), (2.10), which possesses the remarkable approximating property (2.11). Note that if we choose $\gamma=2 \tau^{-1}$ and interpret $w_{n}$ as an approximation at the point $t_{n}=n \tau$, then (2.7) recalls the Cranc-Nikolson difference schema.

3. Parameterized boundary integral equations. We assume that the boundary curve $\Gamma$ is given by a parametric representation

$$
\Gamma=\{x(s): 0 \leq s \leq 2 \pi\},
$$

where $x: \mathbf{R} \rightarrow \mathbf{R}^{2}$ is at least $C^{2}$ and $2 \pi$-periodic with $\left|x^{\prime}(s)\right|>0$ for all $s$. Since the algorithmical treatment of the integral equations (2.17) and (2.19) is very similar to each other we consider only the representation (2.18) of the operator $S_{\gamma}$ and the related boundary integral equations (2.23). These equations take the following parametric form

$$
\begin{aligned}
\frac{1}{2 \pi} \int_{0}^{2 \pi} H_{0}(s, \sigma) \tilde{g}^{(j)}(\sigma) d \sigma & =-\frac{1}{2 \pi} \int_{0}^{2 \pi} \sum_{l=0}^{j-1} H_{j-l}(s, \sigma) \tilde{q}^{(l)}(\sigma) d \sigma, \\
0 & \leq s \leq 2 \pi
\end{aligned}
$$


where

$$
\begin{aligned}
H_{0}(s, \sigma) & =-2 K_{0}(\sqrt{\gamma}|x(s)-x(\sigma)|), \\
H_{n}(s, \sigma) & =-2 \frac{(-2 \gamma)^{n}}{n !} \frac{\partial^{n}}{\partial \gamma^{n}} K_{0}(\sqrt{\gamma}|x(s)-x(\sigma)|) \\
& =-2 \frac{1}{n !}(\sqrt{\gamma}|x(s)-x(\sigma)|)^{n} K_{n}(\sqrt{\gamma}|x(s)-x(\sigma)|), \\
\tilde{q}^{(j)}(\sigma) & =\left|x^{\prime}(\sigma)\right| \tilde{g}^{(j)}(x(\sigma)) .
\end{aligned}
$$

Taking into account the logarithmic singularity of $K_{0}$ we can represent

$$
H_{0}(s, \sigma)=\ln \left(\frac{4}{e} \sin ^{2} \frac{s-\sigma}{2}\right)\left\{1+\sin ^{2} \frac{s-\sigma}{2} H_{0}^{1}(s, \sigma)\right\}+H_{0}^{2}(s, \sigma),
$$

where

$$
\begin{aligned}
H_{0}^{1}(s, \sigma)= & \frac{I_{0}(\sqrt{\gamma}|x(s)-x(\sigma)|)-1}{\sin ^{2}((s-\sigma) / 2)}, \\
H_{0}^{2}(s, \sigma)= & \ln \frac{\gamma e|x(s)-x(\sigma)|^{2}}{16 \sin ^{2}((s-\sigma) / 2)} I_{0}(\sqrt{\gamma}|x(s)-x(\sigma)|) \\
& -2 \sum_{m=0}^{\infty}\left(\frac{r}{2}\right)^{2 m} \frac{\psi(m+1)}{(m !)^{2}}, \quad r=\sqrt{\gamma}|x(s)-x(\sigma)| .
\end{aligned}
$$

Analogously, taking into account the representations (2.25) and (2.31), we see that $\Phi_{n}$ possesses the logarithmic singularity; therefore, we represent for $n \geq 1$,

$$
H_{n}(s, \sigma)=\ln \left(\frac{4}{e} \sin ^{2} \frac{s-\sigma}{2}\right) H_{n}^{1}(s, \sigma)+H_{n}^{2}(s, \sigma)
$$

with

$$
\begin{aligned}
H_{n}^{1}(s, \sigma)= & \frac{(-1)^{n}}{n !} r^{n} I_{n}(r), \\
H_{n}^{2}(s, \sigma)= & \frac{(-1)^{n}}{n !} r^{n} I_{n}(r) \ln \frac{\gamma e|x(s)-x(\sigma)|^{2}}{16 \sin ^{2}((s-\sigma) / 2)} \\
& -\frac{2^{n-1}}{n !} \sum_{m=0}^{n-1}(-1)^{m}\left(\frac{r}{2}\right)^{2 m} \frac{(n-m-1) !}{m !}
\end{aligned}
$$




$$
-\frac{(-1)^{n}}{n ! 2^{n+1}} r^{2 n} \sum_{m=0}^{\infty}\left(\frac{r}{2}\right)^{2 m} \frac{\psi(n+m+1)+\psi(m+1)}{m !(n+m) !} .
$$

Now the functions $H_{i}^{j}, i=0,1, j=1,2$, turn out to be infinitely differentiable. Using the expansions (2.15) and (2.1), we can deduce the diagonal terms

$$
H_{0}^{1}(s, s)=\gamma\left|x^{\prime}(s)\right|^{2}, \quad H_{0}^{2}(s, s)=\ln \frac{\gamma e}{4}\left|x^{\prime}(s)\right|^{2}-2 \gamma .
$$

A discretization of (3.1) can be done by the collocation quadrature method in complete analogy with [3].

4. The heat equation with common boundary condition. Let us consider the problem

$$
\begin{aligned}
\frac{\partial u}{\partial t} & =\Delta u(x, t), \quad(x, t) \in D \times(0, T], \\
u(x, t) & =\phi(x, t), \quad x \in \Gamma, \\
u(x, 0) & =0, \quad x \in D,
\end{aligned}
$$

where, contrary to (2.1), the boundary function depends on both the time and the spatial variables. In order to reduce this problem to the one with time-independent boundary condition, we use the following representation by the Duhamel's integrals

$$
\begin{aligned}
u(x, t) & =\frac{\partial}{\partial t} \int_{0}^{t} U(x, \lambda, t-\lambda) d \lambda \\
& =\int_{0}^{t} \frac{\partial}{\partial t} U(x, \lambda, t-\lambda) d \lambda \\
& =U(x, 0, t)+\left.\int_{0}^{t} \frac{\partial}{\partial \lambda} U\left(x, \lambda, t-\lambda^{\prime}\right)\right|_{\lambda^{\prime}=\lambda} d \lambda,
\end{aligned}
$$

where $U(x, \lambda, t)$ is the solution of the auxiliary problem

$$
\begin{aligned}
\frac{\partial U(x, \lambda, t)}{\partial t} & =\Delta U(x, \lambda, t), \quad(x, t) \in D \times(0, T], \\
U(x, \lambda, t) & =\phi(x, \lambda), \quad x \in \Gamma, \\
U(x, \lambda, 0) & =0, \quad x \in D
\end{aligned}
$$


with the boundary conditions depending on a parameter $\lambda$ but not on the time variable $t$. The last problem can be split up into the stationary case

$$
\begin{aligned}
\Delta V(x, \lambda) & =0, \quad x \in D, \\
V(x, \lambda) & =\phi(x, \lambda), \quad x \in \Gamma,
\end{aligned}
$$

and the nonstationary case with a homogeneous boundary condition

$$
\begin{aligned}
\frac{\partial v(x, \lambda, t)}{\partial t} & =\Delta v(x, \lambda, t), \quad(x, t) \in D \times(0, T], \\
v(x, \lambda, t) & =0, \quad x \in \Gamma, \\
v(x, \lambda, 0) & =-V(x, \lambda), \quad x \in D
\end{aligned}
$$

so that

$$
U(x, \lambda, t)=v(x, \lambda, t)+V(x, \lambda) .
$$

The solution of the initial boundary value problem for the heat equation depends continuously on the given initial and boundary data, see, e.g., $[6$, p. 134] for the maximum norm. For this reason we can use an approximation

$$
\tilde{\Phi}(x, t)=\sum_{i} a_{i}(x) p_{i}(t) \approx \Phi(x, t),
$$

where variables $x$ and $t$ are separated. One of possible constructions with floating accuracy depending on the smoothness of boundary data can be as follows.

Let $t_{0}=0$ and $t_{j}, j=1,2, \ldots, K$ be the roots of the equation

$$
\frac{d}{d t} L_{K+1}^{(0)}(t) \equiv-L_{K}^{(1)}(t)=0
$$

where $L_{n}^{(\alpha)}$ denotes the Laguerre polynomials. Let $\omega_{i}$ be the coefficients of the Gauss-Radau quadrature formula

$$
\begin{aligned}
\int_{0}^{\infty} u(t) e^{-t} d t & \approx \sum_{i=0}^{K} \omega_{i} u\left(t_{i}\right), \\
\omega_{i} & =\frac{1}{(N+1)\left[L_{N+1}^{(0)}\left(t_{i}\right)\right]^{2}},
\end{aligned}
$$


which is exact for $u \in \mathbf{P}_{K}=\{$ all polynomials $p$ with degree $(p) \leq K\}$ [9]. The polynomial

$$
p_{K}(t) \equiv I_{K} u=\sum_{k=0}^{K} a_{k} L_{k}^{(0)}(2 \gamma t)
$$

with

$$
a_{k}=\sum_{i=0}^{K} \omega_{i} u\left(\frac{t_{i}}{2 \gamma}\right) L_{k}^{(0)}\left(t_{i}\right)
$$

is the interpolation polynomial of the function $u(t)$ related to the points $t_{j}, j=0,1, \ldots, K$ which also defines the interpolation operator $I_{K}: C[0, \infty) \rightarrow \mathbf{P}_{K}$. The following holds $[\mathbf{9}]$

$$
\begin{gathered}
\left\|u-I_{K} u\right\|_{\mu, 1} \leq c_{\varepsilon} K^{\mu-((m-1) / 2)}\|u\|_{m, 1-\varepsilon} \\
\forall \varepsilon>0,0 \leq \mu \leq m, m>\frac{1}{2},
\end{gathered}
$$

where

$$
\|v\|_{0, \nu}^{2}=\int_{0}^{\infty} f^{2}(x) e^{-\nu x} d x, \quad\|v\|_{\mu, \nu}^{2}=\sum_{j=0}^{\mu}\left\|\frac{d^{j} v}{d x^{j}}\right\|_{0, \nu}^{2}
$$

Applied to the function $\Phi_{1}(x, t)=e^{\gamma t} \Phi(x, t)$, this approach leads to the approximation

$$
\tilde{\Phi}(x, t)=e^{-\gamma t} I_{K} \Phi_{1}=e^{-\gamma t} \sum_{i=0}^{K} a_{i}(x) L_{i}^{(0)}(2 \gamma t) \approx \Phi(x, t)
$$

with

$$
a_{i}(x)=\sum_{k=0}^{K} \omega_{k} \Phi_{1}\left(x, \frac{t_{k}}{2 \gamma}\right) L_{k}^{(0)}\left(t_{k}\right) .
$$

Due to the linearity of (4.4) and (4.5), one has

$$
V(x, \lambda)=\sum_{i=0}^{K} V_{i}(x, \lambda), \quad v_{i}(x, \lambda, t)=\sum_{i=0}^{K} v_{i}(x, \lambda, t),
$$


where $V_{i}(x, \lambda)$ satisfies the equations

$$
\begin{aligned}
\Delta V_{i}(x, \lambda) & =0, \quad x \in D, \\
V_{i}(x, \lambda) & =e^{-\gamma \lambda} L_{i}^{(0)}(2 \gamma \lambda) a_{i}(x), \quad x \in \Gamma
\end{aligned}
$$

and $v_{i}(x, \lambda, t)$ satisfies the equations

$$
\begin{aligned}
\frac{\partial v_{i}(x, \lambda, t)}{\partial t} & =\Delta v_{i}(x, \lambda, t), \quad(x, t) \in D \times(0, T], \\
v_{i}(x, \lambda, t) & =0, \quad x \in \Gamma, \\
v_{i}(x, \lambda, 0) & =-V_{i}(x, \lambda), \quad x \in D .
\end{aligned}
$$

It is easy to see that

$$
\begin{gathered}
V_{i}(x, \lambda)=e^{-\gamma \lambda} L_{i}^{(0)}(2 \gamma \lambda) \hat{V}_{i}(x), \\
v_{i}(x, \lambda, t)=e^{-\gamma \lambda} L_{i}^{(0)}(2 \gamma \lambda) \hat{v}_{i}(x, t),
\end{gathered}
$$

where the functions $\hat{V}_{i}(x)$ and $\hat{v}_{i}(x, t)$ are the solutions of the problems

$$
\begin{aligned}
\Delta \hat{V}_{i}(x) & =0, \quad x \in D, \\
\hat{V}_{i}(x) & =a_{i}(x), \quad x \in \Gamma
\end{aligned}
$$

and

$$
\begin{aligned}
\frac{\partial \hat{v}_{i}(x, t)}{\partial t} & =\Delta \hat{v}_{i}(x, t), \quad(x, t) \in D \times(0, T], \\
\hat{v}_{i}(x, t) & =0, \quad x \in \Gamma, \\
\hat{v}_{i}(x, 0) & =-\hat{V}_{i}(x), \quad x \in D .
\end{aligned}
$$

Using results of previous sections we can represent

$$
\hat{v}_{i}(x, t)=e^{-\gamma t} \sum_{n=0}^{\infty}(-1)^{n} L_{n}^{(0)}(2 \gamma t) w_{n}^{(i)}(x),
$$

where

$$
\begin{aligned}
w_{0}^{(i)} & =-2 P_{\gamma}\left(\hat{V}_{i}\right), \\
w_{n}^{(i)} & =-2 P_{\gamma}\left[\hat{V}_{i}-\frac{(-2 \gamma)^{n}}{n !} \frac{\partial^{n}}{\partial \gamma^{n}} S_{\gamma}\left(\hat{V}_{i}\right)\right] .
\end{aligned}
$$


Then we get an approximation for $U(x, \lambda, t)$ by

$$
\begin{aligned}
\tilde{U}(x, \lambda, t) & =\sum_{i=0}^{K} e^{-\gamma \lambda} L_{i}^{(0)}(2 \gamma \lambda)\left[\hat{v}_{i}(x, t)+\hat{V}_{i}(x)\right] \\
& =\sum_{i=0}^{K} e^{-\gamma \lambda} L_{i}^{(0)}(2 \gamma \lambda)\left[\sum_{n=0}^{\infty}(-1)^{n} L_{n}^{(0)}(2 \gamma t) w_{n}^{(i)}(x)+\hat{V}_{i}(x)\right] .
\end{aligned}
$$

Using Duhamel's integral we now get the following approximation for $u(x, t)$ :

$$
\begin{aligned}
\tilde{u}_{K}(x, t) & =\int_{0}^{t} \frac{\partial}{\partial t} \tilde{U}(x, \lambda, t-\lambda) d \lambda \\
& =\sum_{i=0}^{K} \sum_{n=0}^{\infty}(-1)^{n} I_{i, n}(t, \gamma) w_{n}^{(i)}(x)+\sum_{i=0}^{K} \hat{V}_{i}(x)
\end{aligned}
$$

and the semi-discrete approximation

$$
\begin{aligned}
\tilde{u}_{K}^{N}(x, t) & =\int_{0}^{t} \frac{\partial}{\partial t} \tilde{U}(x, \lambda, t-\lambda) d \lambda \\
& =\sum_{i=0}^{K} \sum_{n=0}^{N}(-1)^{n} I_{i, n}(t, \gamma) w_{n}^{(i)}(x)+\sum_{i=0}^{K} \hat{V}_{i}(x),
\end{aligned}
$$

where

$$
I_{i, n}(t, \gamma)=\int_{0}^{t} \frac{\partial}{\partial t}\left[e^{-\gamma(t-\lambda)} L_{n}^{(0)}(2 \gamma(t-\lambda))\right] e^{-\gamma \lambda} L_{i}^{(0)}(2 \gamma \lambda) d \lambda .
$$

Using classical results for the Laguerre polynomials, see, e.g., [2], we get

$$
\begin{aligned}
I_{i, n}(t, \gamma)= & -\gamma e^{-\gamma t} \int_{0}^{t} L_{n}^{(0)}(2 \gamma(t-\lambda)) L_{i}^{(0)}(2 \gamma \lambda) d \lambda \\
& +e^{-\gamma t} \frac{\partial}{\partial t} \int_{0}^{t} L_{n}^{(0)}(2 \gamma(t-\lambda)) L_{i}^{(0)}(2 \gamma \lambda) d \lambda-e^{-\gamma t} L_{i}^{(0)}(2 \gamma t)
\end{aligned}
$$




$$
\begin{aligned}
= & {\left[-\frac{1}{2} e^{-\tau / 2} \int_{0}^{\tau} L_{n}^{(0)}(\tau-\xi) L_{i}^{(0)}(\xi) d \xi\right.} \\
& \left.+e^{-\tau / 2} \frac{\partial}{\partial t} \int_{0}^{\tau} L_{n}^{(0)}(\tau-\xi) L_{i}^{(0)}(\xi) d \xi\right]_{\tau=2 \gamma t}-e^{-\gamma t} L_{i}^{(0)}(2 \gamma t) \\
= & \left.-\frac{1}{2} e^{-\tau / 2}\left[L_{n+i}^{(0)}(\tau)-L_{n+i+1}^{(0)}(\tau)\right]+e^{-\tau / 2} L_{n+i}^{(0)}(\tau)\right\}_{\tau=2 \gamma t} \\
& -e^{-\gamma t} L_{i}^{(0)}(2 \gamma t) \\
= & \left.\frac{1}{2} e^{-\gamma t}\left[L_{n+i}^{(0)}(2 \gamma t)+L_{n+i+1}^{(0)}(2 \gamma t)-2 L^{(0)}\right)_{i}(2 \gamma t)\right] .
\end{aligned}
$$

The following algorithm based on the single layer potential performs our approach for the heat equation with a time-dependent boundary condition through boundary integral equations.

\section{Algorithm 2.}

\section{begin}

1. For $i=0,1, \ldots, K$

1.1. Find $a_{i}(x)$ by (4.6) and $\hat{V}_{i}(x)=S_{\gamma}\left(a_{i}\right), x \in \bar{D}$ solving the boundary integral equations

$$
\frac{1}{\pi} \int_{\Gamma} \tilde{g}_{i}(y) K_{0}(\sqrt{\gamma}|x-y|) d s(y)=-a_{i}(x)
$$

and computing

$$
\hat{V}_{i}(x)=-\frac{1}{\pi} \int_{\Gamma} K_{0}(\sqrt{\gamma}|x-y|) \tilde{g}_{i}(y) d s(y)
$$

1.2. $\operatorname{HEBBIE}\left(\hat{V}_{i}, w_{n}^{(i)}\right)$;

2. Find $\tilde{u}_{K}^{N}(x, t)$ by $(4.7)$

end

Note that our approach leads to approximations which are functions of continuous time and spatial arguments. Thus, setting these approximations into the heat equation and using the maximum principle 
one can get a posteriori estimates and construct various feedback algorithms.

Acknowledgments. The authors are grateful to the referees for their valuable suggestions for the improvement of this paper.

\section{REFERENCES}

1. D.Z. Arov, I.P. Gavrilyuk and V.L. Makarov, Representation and approximation of solution of initial value problems for differential equations in Hilbert space based on the Cayley transform, in Elliptic and parabolic problems Proc. of 2nd European Conf., Pont-a-Mousson, June 1994. (C. Bandle, et al., eds.), Pitman Res. Notes Math. Ser. 325 (1995), 40-50.

2. H. Bateman and A. Erdélyi, High transcendental functions, McGraw-Hill Book Company, Inc., New York, 1953.

3. R. Chapko and R. Kress, Rothe's method for the heat equation and boundary integral equations, J. Integral Equations Appl. 9 (1997), 47-69.

4. I.P. Gavrilyuk and V.L. Makarov, Representation and approximation of the solution of an initial value problem for a first order differential equation in Banach space, Z. Anal. Anwendungen 15 (1996), 495-527.

5. http://www.physik.fu-berlin.de/edv_docu/documentation/reduce-3.6p5 /reduce/node181.html

6. R. Kress, Linear integral equations, Springer-Verlag, New York, 1989.

7. N.N. Lebedev, Special functions and their applications, Prentice-Hall, Englewood Cliffs, 1965.

8. C. Lubich and R. Schneider, Time discretizations of parabolic boundary integral equations, Numer. Math. 63 (1992), 455-481.

9. Y. Maday, B. Pernaud-Thomas and H. Vandeven, Une rehabilitation des methodes spectrales de type Laguerre, La Recherche Aerospatiale, Année no. 6 (1985), 353-375.

10. V.L. Makarov and T. Arazmyradov, On the construction of partial solutions of resonance equations, Differentsial'nye Uravneniya 14 (1978), 1255-1261 (in Russian).

11. D. Maki, On constructing distribution function; with applications to Lommel polynomials and Bessel functions, Trans. Amer. Math. Soc. 130 (1968), 81-297.

12. G.N. Watson, A treatise on the theory of Bessel functions, Oxford University Press, Oxford, 1952. 
Berufsakademie Thueringen-Staatliche Studienakademie, Am WartenBERG 2, 99817 Eisenach, Germany

E-mail address: gawrilju@mathematik.uni-leipzig.de, iigawrilju@gmx.de

Institute of Mathematics, National Academy of Sciences, 3 TereschenkivSKA Str., 01601 Kyiv, Ukraine

E-mail address: makarov@imath.kiev.ua 